\title{
Análisis de situaciones familiares relacionadas a la salud mental de niños escolarizados en una comunidad del interior de la Provincia de Córdoba, Argentina
} Analysis of family environments related to primary schoolchildren mental
health in a community located in the Province of Córdoba, Argentina

\author{
María Soledad Burrone ${ }^{1}$, Verónica Gallo², Marcela Lucchese³ ${ }^{3}$, Belén Villace ${ }^{4}$, Mónica Bella ${ }^{5}$,
} Julio Enrique Enders ${ }^{6}$, Ruth Fernández ${ }^{7}$

\begin{abstract}
Resumen
Objetivo: Con la finalidad de establecer un diagnóstico para detectar situaciones de riesgos familiares en niños escolarizados, el objetivo propuesto fue identificar los factores de riesgo vinculados a situaciones familiares que se relacionan con la salud mental, en niños de escuela primaria, de un sector urbano aledaño a la ciudad de Córdoba, en Argentina. Metodología: El instrumento utilizado fue el test 'Cómo es tú-su familia'. Se realizó los análisis exploratorio bivariado y lo factorial. Se entrevistaron a 146 niños escolarizados de quinto y sexto grado del nivel primario. Resultados: La estructura familiar más frecuente fue la biparental y el $45 \%$ de los encuestados tenían estructuras familiares a cargo de mujeres. Más de la mitad de los niños escolarizados refirieron que sus padres nunca, o algunas veces, dedican tiempo para hablar con ellos; los integrantes de su familia no pueden expresarse fácilmente si lo desean y la posibilidad de afrontar los problemas en familia es baja. El 30\% de los niños refirieron haber experimentado alguna situación de estrés. La relación de riesgo con el padre y la madre se asoció a los antecedentes de fumar, peleas, maltratos y emborracharse. Conclusiones: Los resultados de situaciones adversas en el grupo de niños analizados en este trabajo deberían ser tenidos en cuenta para disminuir la vulnerabilidad familiar y favorecer entornos saludables a los niños. Palabras clave: relaciones familiares; salud mental; niños.
\end{abstract}

\begin{abstract}
Objective: In order to establish a diagnosis to identify family risk situations for children in school, the proposed objective was to identify risk factors associated with family situations that relate to mental health in primary schoolchildren from an urban area, adjacent to the city of Cordoba, in Argentina. Methodology: The test 'Cómo es tú-su familia' (What is your family like, in English) was used to interview 146 children attending fifth and sixth grades of primary school. Bivariate exploratory and factorial analyses were performed. Results: The most frequent family structure was the biparental type, with $45 \%$ of interviewees reporting family structures
\end{abstract}

Trabajo realizado en la Universidad Nacional de Córdoba - Córdoba, Argentina.

'Médica, Magister en Salud Pública en la Escuela de Salud Pública, Facultad de Ciencias Médicas, Universidad Nacional de Córdoba - Córdoba, Argentina. ¿Licenciada en Psicología; Maestranda de Salud Pública en la Escuela de Salud Pública, Facultad de Ciencias Médicas, Universidad Nacional de Córdoba - Córdoba, Argentina. ${ }^{3}$ Licenciada en Educación; Magister en Didáctica y Doctoranda en Ciencias de la Salud en la Escuela de Salud Pública, Facultad de Ciencias Médicas, Universidad Nacional de Córdoba - Córdoba, Argentina.

¿Licenciada en Nutrición; Becaria de la Secretaria de Ciencia y Técnica (SECyT); Maestranda en Salud Pública en la Escuela de Salud Pública, Facultad de Ciencias Médicas, Universidad Nacional de Córdoba - Córdoba, Argentina.

${ }^{5}$ Doctora en Medicina y Cirugía; Especialista en Psiquiatría en la Escuela de Salud Pública, Facultad de Ciencias Médicas, Universidad Nacional de Córdoba - Córdoba, Argentina. ${ }^{6}$ Doctor en Ciencias Químicas; Profesor Titular Plenario en la Facultad de Ciencias Médicas, Universidad Nacional de Córdoba - Córdoba, Argentina. `Doctora en Medicina y Cirugía; Magister en Docencia; Profesora Titular en la Escuela de Salud Pública, Facultad de Ciencias Médicas, Universidad Nacional de Córdoba - Córdoba, Argentina.

Dirección para correspondencia: Ruth Fernández - Facultad de Ciencias Médicas - Universidad Nacional de Córdoba - Escuela de Salud Pública - Enrique Barros, s/n - Ciudad Universitaria - Córdoba, Argentina - E-mail: aruthfernandez@gmail.com

Soporto financiero: Fondo Nacional de Ciencia y Tecnología y Proyecto Institucional Salud Investiga (FONCyT).

Conflicto de interese: nada a declarar. 
in which women are in charge. More than 50\% reported that their parents have never or seldom have dedicated time to talk to them, that family members do not find opportunities to express themselves, and that the possibilities of settling problems in family are scarce. In addition, 30\% of children reported having gone through some type of stressful situation. Risk relations with mother and/ or father were associated with smoking, drinking, battering, and quarreling. Conclusions: The adverse conditions detected for this group of children should be taken into account in order to lessen family vulnerability and foster healthy environments for children.

Keywords: family relations; mental health; children.

\section{INTRODUCCIÓN}

Los problemas de comportamiento y salud mental constituyen una de las causas principales de discapacidad en el mundo, con un gran porcentaje de personas que no reciben atención apropiada, a pesar de las propuestas o avances en las estrategias de tratamiento. Así, en las investigaciones se hace referencia a la alta prevalencia y la carga económica generada por los trastornos vinculados a la salud mental ${ }^{1-5}$. Esto también es observado en niños y adolescentes ${ }^{6}$, si bien en América Latina, la magnitud de los trastornos mentales y los aspectos asociados con la salud mental de los niños no han sido suficientemente estudiados ${ }^{7}$. Autores, como Lemos, refieren que el estudio de la salud mental en jóvenes es un área de investigación deficitaria, tanto en el desarrollo de instrumentos y técnicas de valoración, como para el conocimiento de la realidad social y del desarrollo de estrategias asistencia${ }^{l e s}{ }^{8}$, realidad a la que no escapan los antecedentes originados desde Argentina.

Estudios previos en la comunidad Malvinas Argentinas (Provincia de Córdoba) revelan que los Centros de Salud brindan atención efectiva a problemas de salud física, con escasas actividades de promoción de salud y relacionadas con salud mental ${ }^{9}$. Por tal motivo, el equipo de investigación desarrolló diversos trabajos de intervención para fortalecer una red de salud entre la comunidad, las organizaciones y los Centros de Atención Primaria de Salud. Esto requirió efectuar un diagnóstico comunitario sobre la distribución de las necesidades de salud en la comunidad y sus condicionantes, planificando nuevas acciones, y siguiendo las recomendaciones referidas en diversos estudios ${ }^{10,11}$. En función de los trabajos previos y teniendo en cuenta las caracterizaciones sociodemográfica, epidemiológica y del sistema sanitario local, la comunidad mencionada es vulnerable. Por esto, el presente trabajo se orientó a establecer un diagnóstico para detectar situaciones de riesgo familiares a los que están expuestos los niños escolarizados de dicha comunidad.

\section{OBJETIVOS}

Identificar y analizar factores de riesgo vinculados a situaciones familiares que se relacionan con la salud mental, en niños escolarizados en la escuela primaria, de un sector urbano aledaño a la ciudad de Córdoba, en Argentina.

\section{MATERIALES Y MÉTODOS}

Esto fue un estudio observacional y transversal.

\section{Participantes}

La muestra fue comprendida por niños y niñas entre los 10 y 13 años del municipio, que fueron escolarizados en los quintos y sextos grados de escuelas públicas. El tamaño muestral $(n=146)$ fue calculado con un software estadístico $\left(\right.$ InfoStat $^{\circledR}$ ), para lo cual fueron tomados en cuenta el tamaño poblacional y la prevalencia esperada de familias en riesgo, con un nivel de confianza del 95\%.

\section{Instrumentos}

La identificación de los factores de riesgo relacionados a la salud mental se realizó a partir de la aplicación del test "Cómo es tú-su familia"12, lo cual fue elaborado en el Programa de la Organización Panamericana de Salud y Organización Mundial de Salud (OPS/OMS) de Salud del Adolescente y ha sido validado entre otros países en Chile y Brasil. Con formato de opciones múltiples, el test utiliza una perspectiva integradora de enfoques para evaluar la familia. A partir de la aplicación del mismo, fue posible obtener el perfil del funcionamiento familiar, desde la perspectiva de los entrevistados, en base a los puntajes individuales de cada dimensión evaluada en el cuestionario y clasificar la relación parental como de no riesgo, riesgo y alto riesgo. La duración de la entrevista osciló entre los 45 y 60 minutos. El instrumento citado fue validado localmente por el equipo de investigación ${ }^{13}$.

\section{Procedimientos}

Las entrevistas fueron realizadas individualmente por los profesionales de la salud mental, quienes previo a las entrevistas realizaron una jornada educativa brindando la información a los alumnos. Previo a la aplicación se obtuvo la autorización del adulto responsable por el niño y el consentimiento del niño.

\section{Operacionalización de las variables}

Cuadro 1 es un síntesis que incluye las dimensiones evaluadas en el cuestionario y las variables involucradas en cada una de ellas.

\section{Análisis estadístico}

La descripción de las variables se efectuó a partir de las medidas de resumen y el análisis exploratorio, a partir de correspondencia 
Cuadro 1. Dimensiones y variables incorporadas en el cuestionario

\begin{tabular}{|ll|}
\hline Dimensión & \multicolumn{1}{c}{ Variables } \\
Sociodemográficas & $\begin{array}{l}\text { Sexo } \\
\text { Tipo de familia: monoparental, biparental, reconstituida, extensa. }\end{array}$ \\
\hline \multirow{2}{*}{ Relación y comunicación con la familia } & $\begin{array}{l}\text { Relación con el padre, madre y con la familia extensa. } \\
\text { Niveles de satisfacción en: tiempo para hablar, expresión de sentimientos, empatía, apoyo } \\
\text { en dificultades. }\end{array}$ \\
\hline Eventos estresantes & $\begin{array}{l}\text { En el área de la salud: } \\
\text { enfermedad, incapacidad o muerte. } \\
\text { En el área familiar: separación de los padres, violencia en la familia, dificultades económicas }\end{array}$ \\
\hline Conductas de riesgo de violencia & Sufrió de violencia en la familia, violencia con pares. \\
\hline Conductas de riesgo de suicidio & Intentos de suicidio. \\
\hline Conductas de riesgo sexual & Abuso sexual, relaciones sexuales, embarazo no deseado. \\
\hline Conductas de riesgo de consumo de sustancia & Consumo de sustancias tóxicas: tabaco, alcohol, drogas, tranquilizantes. \\
\hline
\end{tabular}

múltiple. Para el análisis bivariado se aplicó el test de $\chi^{2}$ ajustado o el de Fisher, obteniéndose razón de riesgo y los intervalos de confianza (IC). En todos los casos se estableció un nivel de significación del 95\%. El análisis estadístico se efectuó con InfoStat ${ }^{\mathrm{R}}$.

\section{Consideraciones éticas}

El trabajo fue aprobado por el Comité de Ética Local, incluyendo las medidas necesarias para garantizar la confidencialidad de los datos. Así, previo a la aplicación del test se solicitó la autorización del padre/madre o tutor y se le explicó en forma verbal al entrevistado y al adulto responsable acerca del estudio que involucra la investigación, los objetivos del mismo, los procedimientos a seguir, los logros que se esperan del estudio y la garantía de confidencialidad de su identidad. Finalmente se firmó el formulario de consentimiento informado.

\section{RESULTADOS}

El trabajó incluyó a 146 niños escolarizados de los quinto y sexto grados del nivel primario, de los cuales un $49,32 \%$ son mujeres y un 50,68\% son varones. El rango de edad osciló

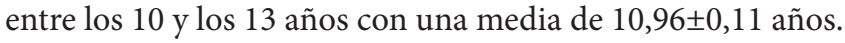

La estructura familiar más frecuente entre los entrevistados fue la biparental (un 54,79\% de los casos), seguida de la monoparental con el $26,03 \%$ y el $19,18 \%$ restante pertenecían a otros tipos.

\section{Relación y comunicación con la familia}

\section{¿Cómo es tu familia?}

Respecto a las características familiares, en el primer ítem, que estudia cohesión familiar, el 47,95\% de los niños refieren que a la familia le gusta pasar el tiempo libre juntos, sin embargo el 73,97\% hace referencia a que los miembros de la familia solamente 'a veces' o 'nunca' pueden expresarse fácilmente si lo desean, siendo este valor significativamente superior a la frecuencia obtenida con las opciones 'siempre' y 'casi siempre' ( $\mathrm{p}<0,0001)$. Esta característica se presentó de igual forma en varones que en mujeres. Este mismo comportamiento se manifestó al preguntar si 'las ideas de los hijos son tenidas en cuenta para afrontar los problemas'. Al respecto sólo el 31,51\% de los niños refieren que casi siempre la familia se une para afrontar los problemas. En cuanto a la pregunta si los miembros de la familia pueden solicitar ayuda entre ellos, se observó que el 53,42\% casi siempre lo hace, y sólo el 5,48\% no lo hacen.

$\mathrm{Al}$ indagar acerca de si se turnan para efectuar las tareas de la casa, el 50,68\% de los casos lo hacen casi siempre, tanto en los varones como en las niñas. En cuanto a si las familias comparten con parientes cercanos los eventos importantes, un $73,97 \%$ refiere hacerlo casi siempre, lo cual es significativamente superior a las otras opciones $(\mathrm{p}<0,0001)$. Finalmente, los niños refirieron mayoritariamente estar satisfechos con la relación con los parientes (el 71,23\%, p<0,003).

\section{Cuando tienes un problema o dificultad ¿a quién buscás} para pedir apoyo o ayuda?

Respecto al apoyo en situaciones de dificultad, la frecuencia detectada se muestra en la Tabla 1.

Cuanto a las características de la comunicación con el padre la frecuencia de respuesta se puede observar en la Tabla 2.

Al indagar cuanto a la percepción de los niños acerca del rol de padre/madre y autoridad en la familia, el $38,4 \%$ refiere que el rol de autoridad está desempeñado por ambos, reflejando un funcionamiento balanceado y democrático. En orden de frecuencia, en el 32,9\% de los casos la autoridad está representada por la madre, y en el 16,4\%, por el padre.

En cuanto a la relación con los padres, se puede observar que los valores de riesgo son similares entre ambos, ubicándose aproximadamente un $28 \%$ de los niños en una relación de riesgo y alto riesgo con los padres (Figura 1). 
Tabla 1. Frecuencia de respuesta respecto a: Cuando tenés un problema o dificultad ¿a quién buscás para pedir apoyo o ayuda?

\begin{tabular}{lccc} 
& Nunca (\%) & Algunas veces (\%) & Casi siempre (\%) \\
Cuando lo necesito cuento con el apoyo de mis padres & 5,48 & 24,66 & 69,86 \\
Cuando lo necesito cuento con el apoyo de alguno de mis hermanos & 21,92 & 50,68 & 27,40 \\
Cuando lo necesito cuento con el apoyo de alguno de mis parientes & 24,66 & 52,05 & 23,29 \\
Cuando lo necesito cuento con el apoyo de alguno de mis amigos & 19,18 & 43,84 & 36,99 \\
Cuando lo necesito cuento con el apoyo de otro adulto & 36,99 & 42,47 & 20,55 \\
\hline
\end{tabular}

Tabla 2. Frecuencia de respuesta de los niños escolarizados respecto a la pregunta: ¿Cómo es la comunicación con tu padre/madre?

\begin{tabular}{|c|c|c|c|c|c|c|}
\hline & \multicolumn{2}{|c|}{ Nunca (\%) } & \multicolumn{2}{|c|}{ Algunas veces (\%) } & \multicolumn{2}{|c|}{ Casi siempre (\%) } \\
\hline & Padre & Madre & Padre & Madre & Padre & Madre \\
\hline Tu padre/madre dedica tiempo cada día para hablar con sus hijos & 27,39 & 6,8 & 39,73 & 32,9 & 32,88 & 60,3 \\
\hline Me gusta la forma en que conversamos con mi padre/madre & 13,7 & 1,4 & 21,92 & 26 & 64,38 & 72,6 \\
\hline Es fácil para expresar mis sentimientos a mi padre/madre & 42,46 & 19,2 & 32,88 & 28,8 & 24,66 & 52,1 \\
\hline Mi padre/madre me entiende & 13,7 & 1,4 & 34,25 & 34,2 & 52,05 & 64,4 \\
\hline Cuando estoy en dificultades, le puedo contar a mi padre/madre & 15,06 & 6,8 & 41,10 & 28,8 & 43,84 & 64,4 \\
\hline
\end{tabular}

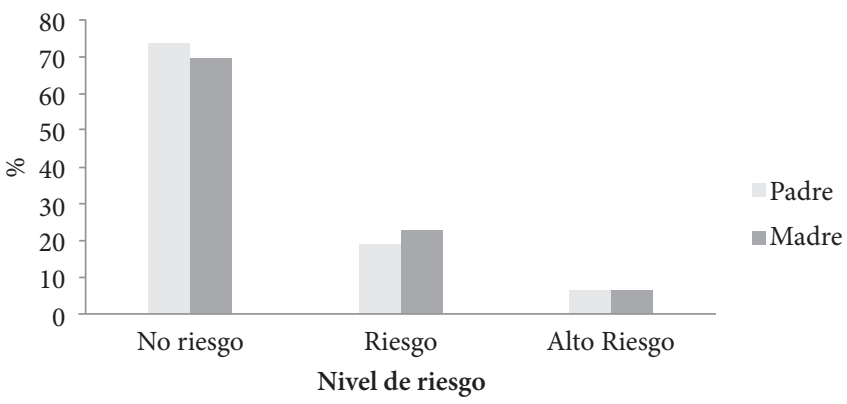

Figura 1. Frecuencia de niveles de riesgo de los niños encuestados en el estudio en relación a la comunicación con los padres

\section{Eventos estresantes en el área de salud y familiar}

En cuanto al análisis de la frecuencia de aparición de eventos estresantes relacionados a los aspectos de las áreas de la salud y familiar, se observó que las situaciones señaladas por los niños como eventos que afectaron a la familia fueron: la muerte de algún miembro de la familia (el 31,5\%), los problemas de dinero en su casa (el 27,4\%), las dificultades de algún miembro de la familia en la escuela por problemas de conducta o rendimiento (el 21,9\%), la enfermedad o incapacidad física de algún integrante de la familia (el 27,4\%), el nacimiento de un hijo de alguien de su familia (el 13,7\%) y los problemas emocionales a nivel familiar (el 17,8\%). Un $12,3 \%$ de los casos refieren como evento estresante la violencia familiar, apareciendo el divorcio de los padres y la mudanza de domicilio con una frecuencia del $11 \%$ de los casos. En un menor orden de frecuencia se registró la fuga del hogar de un integrante de la familia $(9,6 \%$ de los casos), el haber pasado días sin comer por falta de dinero y la situación de que uno de los padres se unió a una nueva pareja $(8,2 \%)$. Por último, se encuentran como situaciones estresantes, el abandono de uno de los padres del hogar y el haberse quedado sin casa presentando frecuencias del 9,6 y $4,1 \%$ respectivamente.
A partir del análisis de los resultados observados en la Tabla 3, se evidenció que, entre el grupo de niños que presentan antecedentes familiares de peleas y de peleas de ellos mismos, estas características están asociadas ( $\mathrm{p}<0,003$; $\mathrm{OR}=2,85$; IC95\% 1,42-5,73). Lo cual implica que el niño con antecedentes de peleas en la familia tiene casi tres veces más posibilidades de involucrarse en peleas que los que no presentan dicho antecedente en su grupo familiar. Esta asociación se observa tanto en el grupo de mujeres como de varones.

Del análisis comparativo entre haber recibido golpes o maltratos y el involucrarse en peleas, se visualizó que el adolescente con maltrato presentaría seis veces más posibilidades de intervenir en peleas que los que no presentan dicho antecedente ( $\mathrm{p}<0,0001$; OR=6,15; IC95\% 2,26-16,68).

Se observó, también, una relación entre el sentirse nervioso y triste todo el tiempo con la presencia de peleas y antecedentes de maltrato $(\mathrm{p}<0,009)$.

Dentro de los comportamientos de riesgo detectados, se encontró con respecto al consumo de sustancias adictivas que el $4,1 \%$ refiere haberse emborrachado. En cuanto al consumo de otro tipo de sustancias adictivas, se observó que el consumo de tabaco, inhalantes y tranquilizantes presenta las mismas frecuencias.

Como factor de riesgo relevante se notó la exposición a una edad temprana de ambientes familiares, en los cuales esta instalado el consumo de sustancias tales como el tabaco, el alcohol, los tranquilizantes y las sustancias ilícitas.

Finalizado el análisis bivariado de todos los ítems de la entrevista, se procedió a realizar un análisis factorial incorporando las variables de riesgo: relación con los padres, participación en peleas, presencia de maltratos, consumo de cigarrillos y antecedentes de emborracharse. Se observa que la asociación entre las variables por cuadrantes se agrupa 
Tabla 3. Distribución de la frecuencia de respuesta respecto a las conductas de riesgo referidas a los niños y a sus familias

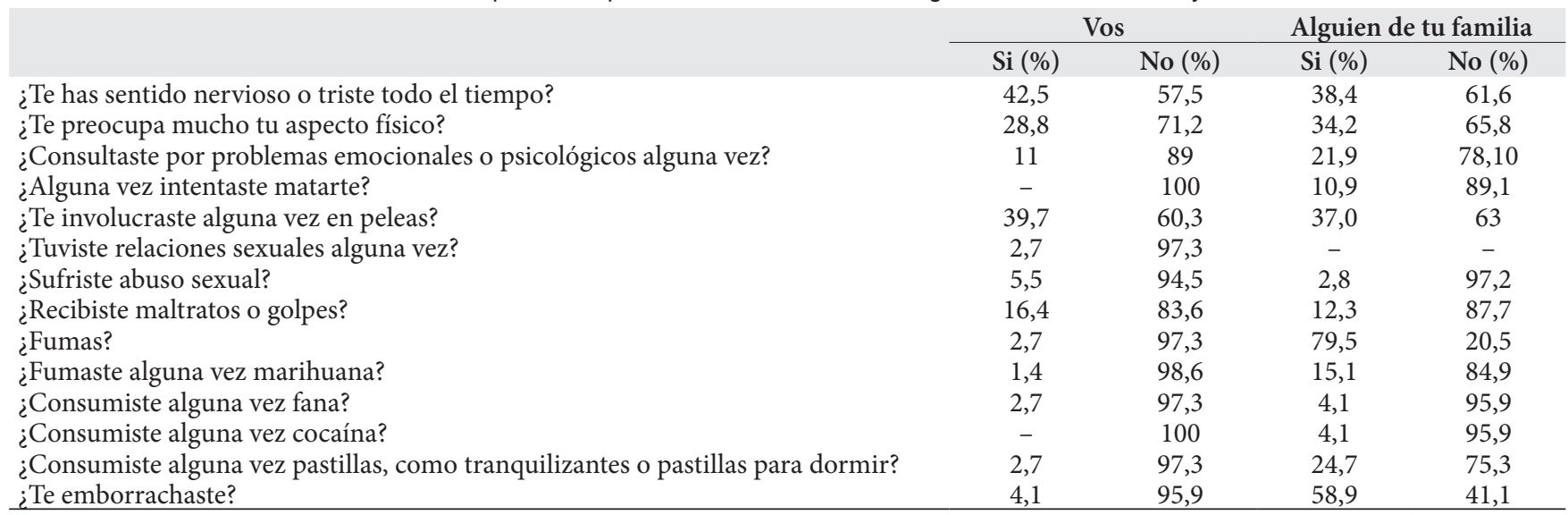

respecto a la relación de riesgo con el padre y la madre y antecedentes de fumar, de peleas, maltratos y emborracharse. Mientras que la ausencia de todos estos factores se encuentra agrupada a la situación de no riesgo respecto a la relación con el padre y la madre (Figura 2).

\section{DISCUSIÓN}

Desde hace varias décadas, diferentes investigadores refieren que hay una pandemia creciente de trastornos mentales y proponen, como una necesidad prioritaria, realizar evaluaciones epidemiológicas de los trastornos mentales, en función de los cambios demográficos y que se producen en la estratificación de la población ${ }^{14,15}$. Esta necesidad se evidencia en todos los grupos etarios. Se observa que las conductas de riesgo en esta población, como intentos de suicidio, consumo de drogas licitas e ilícitas, violencia intrafamiliar y entre pares, conductas sexuales de riesgo entre otras, son causas importantes de discapacidad y años de vida perdida ${ }^{16}$. La programación de la salud mental en niños y jóvenes debe basarse en el conocimiento de la población que será sujeto y objeto de las actividades y en la determinación de sus necesidades generales y de salud. Las acciones a efectuar deben ser el análisis respecto a la forma en que estos factores inciden en la salud mental y en las técnicas de intervención existentes, y sus posibilidades de utilización en el área, tomando en cuenta tanto la aceptación de la comunidad como los recursos técnicos y financieros ${ }^{17}$. En este marco y a partir de esta investigación, se identificó que el $45 \%$ de los niños encuestados tienen una estructura familiar a cargo de mujeres. Estos resultados son coincidentes con los manifestados por la Comisión Económica para América Latina y el Caribe (CEPAL), que ha estimado que aproximadamente uno de cada cinco hogares en Latinoamérica está encabezado por una mujer ${ }^{18}$. Antecedentes similares han sido informados en trabajos efectuados en Costa Rica, El Salvador y en Trinidad y Tobago ${ }^{19-21}$.
El funcionamiento familiar se basa, entre otros factores, en la cohesión, la adaptabilidad y la comunicación de los miembros que forman la familia ${ }^{22-24}$. Asumiendo que la adaptabilidad es definida como la habilidad del sistema familiar para modificar las estructuras de poder, los roles y las reglas de relación, en respuesta al desarrollo evolutivo vital de la familia o en respuesta al estrés provocado por diversas situaciones concretas, este factor se ve favorecido por la comunicación familiar ${ }^{25}$. En función de lo expresado, en el presente estudio se mostró que más de la mitad de los niños escolarizados refirieron que sus padres nunca, o algunas veces, dedican tiempo para hablar con ellos, los integrantes de su familia no pueden expresarse fácilmente si lo desean y la posibilidad de afrontar los problemas en familia es baja. Sin embargo, los niños refieren en más de la mitad de los casos que los miembros de las familias solicitan ayuda entre ellos y comparten los eventos con sus parientes cercanos, percibiendo en la mayoría de los casos fuentes de apoyo familiar.

En cuanto a los eventos estresantes, aproximadamente el $30 \%$ de los entrevistados refirieron haber experimentado alguna situación de estrés en su entorno familiar. Abordar acciones preventivas en este grupo será relevante para los responsables de la salud en la comunidad ya que, como mencionan algunos autores, si los eventos de vida negativos ocurren antes de la edad de 18 años, tienen un mayor impacto en el desarrollo de deficiencias cognitivas, predisponiendo a los adolescentes a una conducta de riesgo antes o durante la adultez joven ${ }^{26,27}$.

Otros factores intervinientes en los niveles de adaptabilidad familiar son las situaciones difíciles vividas en la familia. Al respecto, en el grupo estudiado, hubo una asociación entre los niños entrevistados que sufrieron maltrato y antecedentes de haber participado en peleas y se identificó como factor de riesgo relevante los ambientes familiares en los cuales está instalado el consumo de sustancias, tales como el tabaco, el alcohol, los tranquilizantes y las sustancias ilícitas. Es de destacar que se encontró una relación entre el maltrato físico y/o verbal, la violencia intrafamiliar y el alcoholismo en alguno de los progenitores, y las 


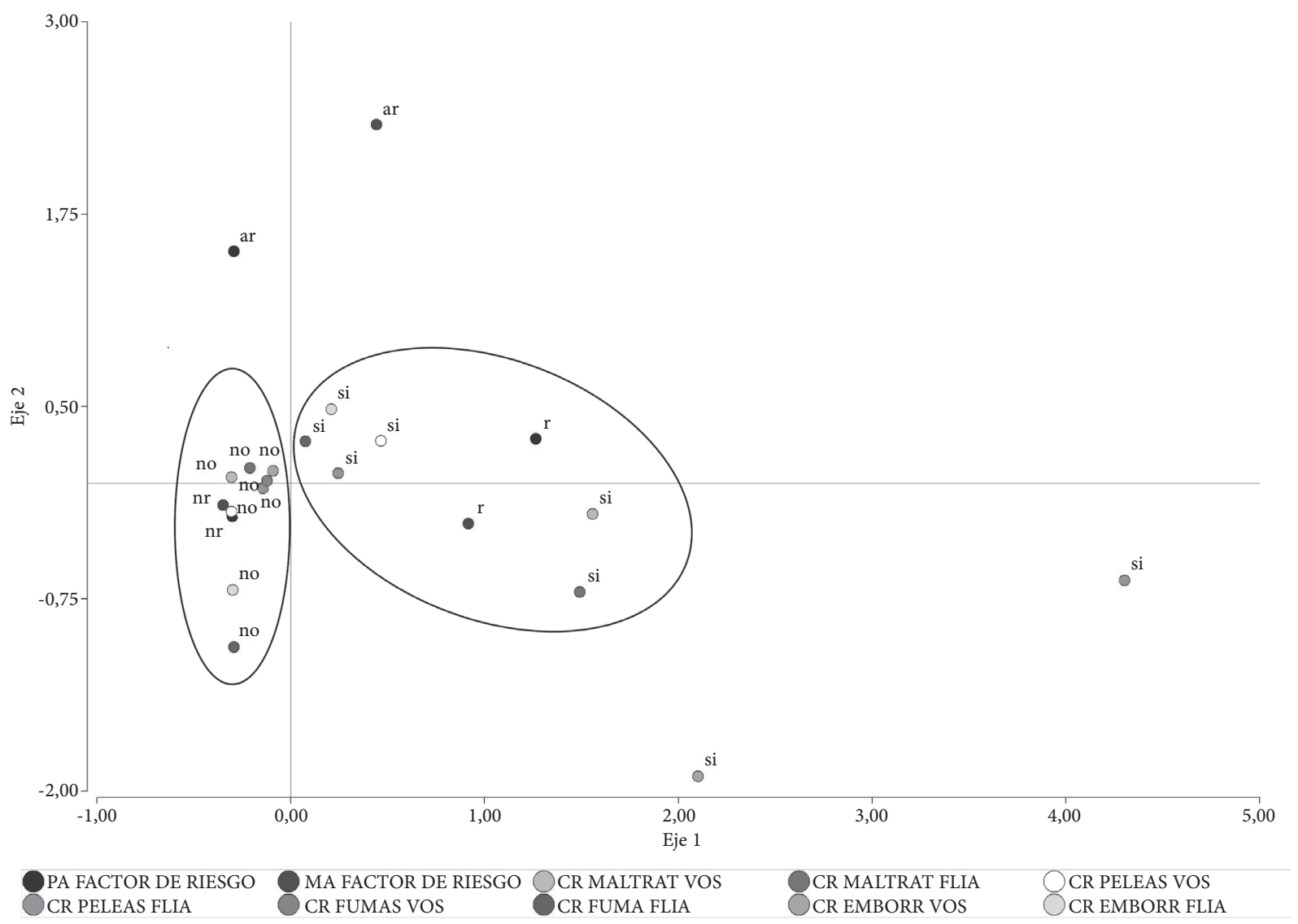

Figura 2. Agrupación de las variables: relación de riesgo con el padre y con la madre, antecedentes de peleas, maltratos, de fumar y emborracharse

conductas de abuso y/o adicción en los niños. Esta asociación de riesgo para desarrollar conductas de abuso y/o adicción de drogas es coincidente a lo presentado por otras investigaciones ${ }^{28,29}$. Obano Solano y Sáenz Rojas refieren en su trabajo que en aquellos estudiantes que reconocieron consumir alguna droga, el $43,4 \%$ tuvo como modelo a alguien de la familia ${ }^{30}$.

En síntesis, los resultados de situaciones adversas en el grupo de niños analizados en este trabajo deberían ser tenidos en cuenta para disminuir la vulnerabilidad familiar y favorecer entornos saludables a los niños, considerando las recomendaciones de los organismos internacionales, respecto a generar estrategias de promoción de la salud dentro del contexto de la familia y de la comunidad ${ }^{20}$.

\section{RECONOCIMIENTO}

Profesionales que colaboraron con la ejecución de las entrevistas: Escutti C., Menel C. y Vila M. y López de Neira M.S.

\section{REFERENCIAS}

1. Dewa CS, Lin E. Chronic physical illness, psychiatric disorders and disability in the workplace. Soc Sci Med. 2000;51(1):41-50.

2. Ustün TB, Ayuso-Mateos JL, Chatterii S, Mathers C, Murray CJ. Global burden of depressive disorders in the year 2000. Br J Psychiatry. 2004;184:386-92.

3. Alvarado Muñoz R, Numhauser JT. Prevalencia de cuadros depresivos y factores asociados en mujeres adultas de la Isla de Chiloé. Rev Chil Salud Pública. 2004;8(1):7-17.

4. Osada JL, Arriola-Quiroz I. Mental health focus. Rev Med Chile. 2009;137:1532-7.

5. OPS/OMS. Boletín oficial de Situación de Salud en las Américas. Indicadores Básicos; 2007. [cited 2012 Nov 08]. Available from: http:// www.paho.org/home_spa.htm
6. Caraveo-Anduaga JJ, Colmenares-Bermúdez E, Martínez-Vélez NA. Síntomas, percepción y demanda de atención en salud mental en niños y adolescentes de la Ciudad de México. Rev Salud Pública México. 2002;44(6):492-8.

7. Restrepo. Psiquiatría en salud mental de niños y adolescentes: una necesidad. Rev Colombiana Psiqu. 2005;34(3):339-41.

8. Lemos S, Fidalgo A, Calvo P, Menéndez P. Salud mental de los adolescentes asturianos. Psicothema. 1992;4(1):21-48.

9. Burrone MS, Fernández AR, Acevedo GE, Lucchese MS, Lopez de Neira MJ, Dell Inocenti P, et al. Análisis de la estrategia de atención primaria de salud en el municipio de Malvinas Argentinas: un abordaje cuanticualitativo. Rev Salud Pública. 2007;11(2):7-22. 
10. OMS. Informe servicio comunitario en salud mental: reducción de la exclusión social. 2007. [cited 2012 Nov 08]. Available from: http://www.who.int/es/

11. García López R. Salud mental comunitaria: ¿una tarea interdisciplinaria? Cuad Trabajo Social. 2004;17:273-87.

12. OMS. Familia y adolescencia: Indicadores de salud. Washington; 1996. [cited 2012 Nov 08]. Available from: http://www.who.int/es/

13. Bella ME, Fernández AR, Acevedo G, Willington JM. Análisis Sociodemográfico y psicopatológico en intentos de suicidio infantojuveniles. VERTEX Rev Arg Psiqu. 2008;19:10-6.

14. Kramer M. The rising pandemic of mental disorders and associated chronic disease and disabilities. Acta Pshychiatric Scand. 1980;62:285.

15. Levav I, Lima BR, Somoza Lennon M, Kramer M, González R. Salud Mental para todos en América Latina y el Caribe. Bol Oficin Sanit Panam. 1989;107:196-219.

16. OMS. Informe sobre la Salud en el mundo. Salud Mental: Nuevos conocimientos, nuevas esperanzas. 2001. [cited 2012 Nov 08]. Available from: http://www.who.int/es/

17. Arroyo Sucre J. Programas de Salud Mental del Niño en el Nivel Comunitario. In: Levav I. Temas de Salud Mental en la Comunidad. Serie Paltex para ejecutores de programas de salud; n. 19; 1992. 205 p.

18. Comisión Económica para América Latina y El Caribe (CEPAL). Anuario Estadístico de América Latina y El Caribe 1999. Nueva York: Naciones Unidas; 2000.

19. Halcón L, Beuhring T, Blum R. A portrait of adolescent health in The Caribbean. Minneapolis: Universidad de Minnesota, OPS; 2000.

20. Maddaleno M, Morello P, Infante-Espínola F. Salud y desarrollo de adolescentes y jóvenes en Latinoamérica y El Caribe: desafíos para la próxima década. Salud Pública Mex. 2003;45(1):132-9.

21. Schutt-Aine J, Maddaleno M. Salud sexual y desarrollo de adolescentes y jóvenes en las Américas: implicaciones para programas y políticas. Washington, DC: OPS/OMS; 2002.
22. Zegers B, Larraín ME, Polaino-Lorente A, Trapp A, Diez I. Validity and reliability of the Olson, Russel and Sprenkle Family Cohesión and Adaptability Scale in diagnosing family functioning in the Chilean population. Rev Chil Neuro-Psiquiat. 2003;41(1):39-54.

23. López Larrosa S. El FACES II en la evaluación de la cohesión y la adaptabilidad familiar. Psicothema. 2002;14(1):159-66.

24. Olson DH, McCubbin HI, Barnes H, Larsen A, Muxen M, Wilson M. Family Inventories. St. Paul, Minnesota: Family Social Science, University of Minnesota; 1985.

25. González-Pienda J, Núñez J, Álvarez L, Roces C, González-Pumariega S, González P, et al. Adaptabilidad y cohesión familiar, implicación parental en conductas autorregulatorias, autoconcepto del estudiante y rendimiento académico. Psicothema. 2003;15(3):471-7.

26. Yang B, Clum GA. Effects of early negative life experiences on cognitive functioning and risk for suicide: A review. Clin Psychol Rev. 1996;16(3):177-95.

27. Rosselló J, Berríos Hernández M. Ideación Suicida, Depresión, Actitudes Disfuncionales, Eventos de Vida Estresantes y Autoestima en una Muestra de Adolescentes Puertorriqueños/as. Rev Interamericana Psicol 2004;38(2):295-302.

28. Resnick MD, Bearman PS, Blum RW, Bauman KE, Harris KM, Jones J, et al. Protecting adolescents from harm. Findings from the national longitudinal study on adolescent health. JAMA. 1997;278:823-32.

29. Rees R, Valenzuela A. Características individuales y de la estructura familiar de un grupo de adolescentes abusadores de alcohol y/o marihuana. Rev Chil Neuro-psiquiatr. 2003;41(3):173-86.

30. Obando Solano P, Sáenz Rojas MA. Percepción, consumo y factores asociados con el fenómeno droga en población escolar de Heredia, Costa Rica. Adicciones. 2000;12(1):127-35.

Recibido en: 22/07/2012

Aprobado en: 07/10/2012 